\title{
Desempenho agronômico de novas linhagens de amendoim no Cerrado
}

Submetido - 07 jul. $2020 \quad$ Aprovado - 07 ago. $2020 \quad$ Publicado - 14 out. 2020
dol
http://dx.doi.org/10.17648/sas.v1i1.8

\begin{abstract}
Jair Heuert (iD
Programa de Melhoramento do Amendoim - Embrapa, Santo Antônio de Goiás, GO, jair.heuert@embrapa.br.
\end{abstract}

\section{Lúcio Lima Rodrigues ${ }^{(D)}$}

Discente de Engenharia Básica da UNIP, Goiânia, GO, luciolimarodrigues@gmail.com.

\section{Kennedy Brunno de Brito Martins (DD}

Programa de Melhoramento do Amendoim - Embrapa, Santo Antônio de Goiás, GO, kennedybrunno22@gmail.com.

Maxuel Fellipe Nunes Xavier (i)

Discente de Agronomia do IFMT Campus São Vicente - Centro de Referência de Campo Verde, Campo Verde, MT, maxuelfellipe90@gmail.com.

Taís de Moraes Falleiro Suassuna (iD)

Programa de Melhoramento do Amendoim - Embrapa, Santo Antônio de Goiás, GO, tais.suassuna@embrapa.br.

\section{RESUMO}

Este trabalho teve como objetivo de avaliar o desempenho agronômico de novas linhagens de amendoim no Cerrado. O experimento foi conduzido na Embrapa Arroz e Feijão, localizada em Santo Antônio de Goiás-GO, na safra 2019/20. U utilizado o delineamento experimental em blocos casualizados, com quatro repetições e quatorze tratamentos, sendo dez linhagens: 171253 OL, 18-1973 OL, 18-2010 OL, 18-2055 OL, 18-2087 OL, 18-2101 OL, 18-2133 OL, 18-2136 OL, 18-2177 OL, 18-2182 OL e quatro cultivares comerciais: BRS 421 OL, BRS 423 OL, BRS 425 OL e Granoleico. As parcelas foram formadas por duas linhas de três metros de comprimento e espaçamento entre linhas de noventa centímetros. Foram avaliados severidade de mancha preta, massa de 100 grãos e produtividade de vagens. As menores severidades de mancha preta foram obtidas nos genótipos BRS 421 OL, BRS 425 OL e 18-2133 OL. Os genótipos mais produtivos foram BRS 423 OL, BRS 421 OL, 18-2133 OL, 18-2010 OL, BRS 425 OL e 17-1253 OL.

Palavras-chave: Arachis hypogaea L.; Programa de Melhoramento; Produtividade.

\section{Agronomic performance of new peanut strains in the Cerrado}

\begin{abstract}
This work aimed to evaluate the agronomic performance of new peanut strains in the Cerrado. The experiment was conducted at Embrapa Arroz e Feijão, located in Santo Antônio de GoiásGO, in the 2019/20 harvest. A randomized block design was used, with four replications and fourteen treatments, ten lines: 17-1253 OL, 18-1973 OL, 18-2010 OL, 18-2055 OL, 18-2087 OL, 182101 OL, 18-2133 OL, 18-2136 OL, 18-2177 OL, 18-2182 OL and four commercial cultivars: BRS $421 \mathrm{OL}, B R S 423 \mathrm{OL}, B R S 425 \mathrm{OL}$ and Granoleico. The plots were formed by two lines of three meters in length and spacing between lines of ninety centimeters. Severity of black spot, mass of 100 grains and pod yield were evaluated. The lowest severity of black spot was obtained in the genotypes BRS 421 OL, BRS $425 \mathrm{OL}$ and 18-2133 OL. The most productive genotypes were BRS 423 OL, BRS 421 OL, 18-2133 OL, 18-2010 OL, BRS 425 OL and 17-1253 OL.
\end{abstract}

Keywords: Arachis hypogaea L.; Improvement Program; Productivity. 


\title{
Rendimiento agronómico de nuevas cepas de maní en el Cerrado
}

\section{RESUMEN}

\begin{abstract}
Este trabajo tuvo como objetivo evaluar el rendimiento agronómico de las nuevas cepas de maní en el Cerrado. El experimento se realizó en Embrapa Arroz e Feijão, ubicado en Santo Antônio de Goiás-GO, en la cosecha 2019/20. Se utilizó un diseño de bloques al azar, con cuatro repeticiones y catorce tratamientos, diez líneas: 17-1253 OL, 18-1973 OL, 18-2010 OL, 182055 OL, 18-2087 OL, 18-2101 OL, 18-2133 OL, 18-2136 OL, 18-2177 OL, 18-2182 OL y cuatro cultivares comerciales: BRS 421 OL, BRS 423 OL, BRS 425 OL y Granoleico. Las parcelas estaban formadas por dos líneas de tres metros de longitud y espaciadas entre líneas de noventa centímetros. Se evaluaron la gravedad de la mancha negra, la masa de 100 granos y el rendimiento de la vaina. La menor severidad de la mancha negra se obtuvo en los genotipos BRS 421 OL, BRS 425 OL y 18-2133 OL. Los genotipos más productivos fueron BRS 423 OL, BRS 421 OL, 18-2133 OL, 18-2010 OL, BRS 425 OL y 17-1253 OL.
\end{abstract}

Palabras clave: Arachis hypogaea L.; Programa de Mejoramiento; Productividad.

\section{Introdução}

O amendoim (Arachis hypogaea L.) é uma importante fonte de proteínas e óleo, amplamente utilizada no processamento de alimentos em diversos países. De acordo com o Departamento de Agricultura dos Estados Unidos (2020), a produção mundial de amendoim foi de aproximadamente 45,4 milhões de toneladas na safra 2019/20. Os maiores produtores mundiais dessa oleaginosa são os países da China (38,6\%), Índia (13,7\%), Nigéria (7,7\%), Estados Unidos (5,5\%), Sudão (4,0\%), Myanmar (3,0\%) e Argentina (2,6\%), sendo o Brasil o décimo quarto colocado com uma participação de 1,2\%.

A pesquisa para o desenvolvimento de novas cultivares, pode ser considerada um processo contínuo e fundamental para o sucesso da cadeia produtiva. O melhoramento genético permite gerar novas cultivares mais produtivas, com melhor adaptação regional, ciclo adequado, hábito de crescimento adaptado a colheita mecanizada, resistência a pragas, doenças e ao estresse hídrico. Além disso, as características mercadológicas dos grãos precisam estar em consonância com às exigências do mercado consumidor, como a qualidade visual, nutricional, química, sensorial, formato e granulometria (VIZEU, 2019). 
No Registro Nacional de Cultivares (RNC) do Ministério da Agricultura foi incluído pela Embrapa, três novos cultivares de amendoim do tipo rasteiro, com alto teor de ácido oleico e tipo de grãos no padrão "runner", que atendem aos mercados mais exigentes. As cultivares BRS 421 OL e BRS 423 OL, são adaptadas ao bioma Cerrado no Brasil Central (Mato Grosso do Sul, Mato Grosso, Goiás e Tocantins) (SUASSUNA et al., 2020). A BRS $425 \mathrm{OL}$ possui uma ampla adaptação as principais regiões produtoras de amendoim do Brasil (SUASSUNA et al., 2019). O presente trabalho teve como objetivo avaliar desempenho agronômico de novas linhagens de amendoim no Cerrado.

\section{Material e métodos}

O experimento foi instalado na Fazenda Capivara da Embrapa Arroz e Feijão, localizada no município de Santo Antônio de Goiás-GO, na época propicia para cultivo de amendoim nesta região (HEUERT et al., 2018), no dia 2 de dezembro de 2019. O delineamento experimental foi disposto em blocos casualizados, com quatro repetições e quatorze tratamentos, sendo dez linhagens: 17-1253 OL, 18-1973 OL, 18-2010 OL, 18-2055 OL, 18-2087 OL, 18-2101 OL, 18-2133 OL, 18-2136 OL, 18-2177 OL, 18-2182 OL e quatro cultivares comerciais: BRS $421 \mathrm{OL}$, BRS $423 \mathrm{OL}$, BRS $425 \mathrm{OL}$ e Granoleico. As parcelas foram formadas por duas linhas de três metros de comprimento, com espaçamento de noventa centímetros, intervalo de três metros entre parcelas, e estande médio de 15 plantas por metro.

No campo experimental, foi avaliado severidade da mancha preta aos 120 dias após semeadura, usando a escala diagramática, com notas de 1-9, proposta por SUBRAHMANYAM et al. (1982). O experimento foi invertido no dia 27 de abril de 2020, usando arrancador simples da marca Agromérica $\AA^{\circledR}$ e após o período de secagem no campo foram colhidos para determinar a massa de 100 grãos $(\mathrm{g})$ e a produtividade de vagens $\left(\mathrm{kg} / \mathrm{ha}^{-1}\right)$. A análise de variância foi realizada usando o programa SISVAR 5.6 e o teste de médias empregado foi o Scott Knott, a 5\% de probabilidade (FERREIRA, 2019). 


\section{Resultados e discussão}

O experimento apresentou elevada produtividade e diferença significativa para todas as variáveis analisadas (Tabela 1). A severidade média do experimento foi de 7,8 . As menores notas de severidade foram observadas nos genótipos BRS 421 OL $(6,8)$, BRS 425 OL $(7,2)$ e 18-2133 OL $(7,2)$. Estes resultados são semelhantes aos relatados por Heuert et al. (2018), que observaram menores severidades nos genótipos BRS 421 OL (13-374 OL) e BRS 425 OL (13-425 OL) nas condições de Cristianópolis e Santo Antônio de Goiás, em Goiás. No entanto, as maiores severidades foram observadas nos genótipos 18-2182 OL $(7,9)$, Granoleico $(8,1)$, 171253 OL $(8,1), 18-2055$ OL (8,2), 18-1973 OL $(8,3)$ e 18-2101 OL $(8,6)$, que apresentaram desfolha intensa no terço médio das plantas. Nesse sentido, Martins et al. (2019), observaram maiores notas de severidade nos genótipos Granoleico $(7,8)$ e 18-2101 OL $(8,4)$ em Santo Antônio de GoiásGO. Mesmo estando entre os genótipos com maiores severidades, as linhagens 17-1253 OL e 18-2101 OL produziram mais de $6.000 \mathrm{~kg} / \mathrm{ha}^{-1} \mathrm{de}$ amendoim em vagens.

Quanto a massa de 100 grãos, observa-se diversidade na granulometria dos diferentes genótipos testados. Verifica-se as maiores massas, ou seja, os maiores grãos com os genótipos BRS 421 OL $(86,7 \mathrm{~g})$, 18-1973 OL (81,4 g) e 18-2010 OL (80,0 g). Martins et al. (2018 e 2019) observou o genótipo BRS $421 \mathrm{OL}$ (13-374 OL) entre as maiores massas. Os genótipos 17-1253 OL (78,5 g), Granoleico (78,0 g), 18-2177 OL (77,9 g), 18-2101 OL $(76,8 \mathrm{~g})$ e 18-2133 OL $(76,6 \mathrm{~g})$, resultaram em grãos de tamanho intermediário. Ribeiro et al. (2018), observaram no genótipo Granoleico uma massa de $77,7 \mathrm{~g}$, semelhante ao presente estudo. As menores massas foram obtidas nos genótipos 18-2136 OL (75,7 g), BRS $423 \mathrm{OL}(75,5 \mathrm{~g})$, BRS $425 \mathrm{OL}(75,3 \mathrm{~g}), 18-2087 \mathrm{OL}(75,1 \mathrm{~g}), 18-2182 \mathrm{OL}$ $(74,6 \mathrm{~g})$ e $18-2055 \mathrm{OL}(74,5 \mathrm{~g})$. Martins et al. (2018) obtiveram massa semelhante com o BRS $425 \mathrm{OL}(75,6 \mathrm{~g})$, sendo uma das menores do referido estudo. 
Tabela 1. Severidade de mancha preta (notas), massa de 100 grãos (g) e produtividade em vagens $\left(\mathrm{kg} / \mathrm{ha}^{-1}\right.$ e sacas/alqueire $\left.{ }^{-1}\right)$ em função de diferentes genótipos de amendoim. Santo Antônio de Goiás-GO, 2019/20.

\begin{tabular}{|c|c|c|c|c|}
\hline \multirow{2}{*}{ Genótipos } & \multirow{2}{*}{$\begin{array}{c}\text { Severidade de } \\
\text { mancha preta } \\
\text { (notas) }\end{array}$} & \multirow{2}{*}{$\begin{array}{c}\text { Massa de } \\
100 \text { grãos } \\
(g)\end{array}$} & \multicolumn{2}{|c|}{ Produtividade em vagens } \\
\hline & & & $\left(\mathrm{kg} / \mathrm{ha}^{-1}\right)$ & (sacas/alqueire $^{-1}$ ) \\
\hline BRS $423 \mathrm{OL}$ & $7,5 \mathrm{c}$ & $75,5 d$ & $8.360,6 \mathrm{a}$ & 809,3 \\
\hline BRS $421 \mathrm{OL}$ & $6,8 \mathrm{a}$ & $86,7 \mathrm{a}$ & $8.001,8 \mathrm{a}$ & 774,6 \\
\hline $18-2133 \mathrm{OL}$ & $7,2 \mathrm{~b}$ & $76,6 \mathrm{c}$ & $7.871,8 \mathrm{a}$ & 762,0 \\
\hline $18-2010$ OL & $7,7 \mathrm{c}$ & $80,0 \mathrm{~b}$ & $7.785,4 \mathrm{a}$ & 753,6 \\
\hline BRS $425 \mathrm{OL}$ & $7,2 \mathrm{~b}$ & $75,3 d$ & $7.776,1 \mathrm{a}$ & 752,7 \\
\hline 17-1253 OL & $8,2 d$ & $78,5 \mathrm{c}$ & $6.789,4 \mathrm{a}$ & 657,2 \\
\hline $18-2101 \mathrm{OL}$ & $8,6 \mathrm{~d}$ & $76,8 \mathrm{c}$ & $6.141 .2 \mathrm{~b}$ & 594,5 \\
\hline 18-2055 OL & $8,3 d$ & $74,5 d$ & $5.966,4 \mathrm{~b}$ & 577,5 \\
\hline $18-2136$ OL & $7,4 \mathrm{c}$ & $75,7 d$ & $5.775,7 \mathrm{~b}$ & 599,1 \\
\hline $18-2177$ OL & $7,4 \mathrm{c}$ & $77,9 \mathrm{c}$ & $5.581,3 b$ & 540,3 \\
\hline 18-1973 OL & $8,3 d$ & $81,4 \mathrm{~b}$ & $5.185,5 b$ & 501,9 \\
\hline Granoleico & $8,1 \mathrm{~d}$ & $78,0 \mathrm{c}$ & $4.829,5 b$ & 467,5 \\
\hline 18-2182 OL & $7,9 \mathrm{~d}$ & $74,6 d$ & $4.657,8 \mathrm{~b}$ & 450,9 \\
\hline 18-2087 OL & $7,5 \mathrm{c}$ & $75,1 d$ & $4.632,3 \mathrm{~b}$ & 448,5 \\
\hline Média & 7,7 & 77,6 & $6.382,5$ & 610,63 \\
\hline C.V. (\%) & 3,3 & 1,6 & 15,0 & - \\
\hline $\mathrm{Pr}>\mathrm{Fc}$ & $>0,0001^{*}$ & $>0,0001^{*}$ & $>0,0001^{*}$ & - \\
\hline 要 & $5 \%$ de & $\begin{array}{l}\text { ade pelo } \\
\text { şão. }\end{array}$ & $\mathrm{e}$ de $\mathrm{Sc}$ & Knott; ns - n \\
\hline
\end{tabular}

As maiores produtividades em vagens foram observadas nos genótipos BRS $423 \mathrm{OL}\left(8.360,6 \mathrm{~kg} / \mathrm{ha}^{-1}\right)$, BRS $421 \mathrm{OL}\left(8.001,8 \mathrm{~kg} / \mathrm{ha}^{-1}\right), 18-$ $2133 \mathrm{OL}\left(7.871,8 \mathrm{~kg} / \mathrm{ha}^{-1}\right), 18-2010 \mathrm{OL}\left(7.785,4 \mathrm{~kg} / \mathrm{ha}^{-1}\right)$, BRS $425 \mathrm{OL}$ $\left(7.776,1 \mathrm{~kg} / \mathrm{ha}^{-1}\right)$ e $17-1253 \mathrm{OL}\left(6.789,4 \mathrm{~kg} / \mathrm{ha}^{-1}\right)$. Dentre as cultivares da Embrapa, verifica-se que a BRS $425 \mathrm{OL}$ foi menos produtiva que BRS 423 OL e BRS 421 OL, nas condições de Santo Antônio de Goiás-GO. Corroborando com Suassuna et al. (2020), que descrevem o BRS $423 \mathrm{OL}$ e BRS 421 OL com adaptação ao bioma Cerrado. Os demais genótipos obtiveram produtividades inferiores a média geral do experimento, de 6.280 $\mathrm{kg} / \mathrm{ha}^{-1}$. No entanto, os genótipos 19-2182 OL $\left(4.657,8 \mathrm{~kg} / \mathrm{ha}^{-1}\right)$ e $18-2087$ OL $\left(4.632,3 \mathrm{~kg} / \mathrm{ha}^{-1}\right)$ foram menos produtivos que a cultivar Granoleico $\left(4.829,5 \mathrm{~kg} / \mathrm{ha}^{-1}\right)$. Constata-se que dentre as linhagens, somente a $18-2133$ 
OL obteve uma das maiores produtividades estando entre as menores notas de severidade de mancha preta.

\section{Conclusões}

As menores severidades de mancha preta foram obtidas nos genótipos BRS 421 OL, BRS 425 OL e 18-2133 OL. Os genótipos mais produtivos foram BRS $423 \mathrm{OL}$, BRS $421 \mathrm{OL}, 18-2133 \mathrm{OL}, 18-2010 \mathrm{OL}$, BRS $425 \mathrm{OL}$ e $17-1253 \mathrm{OL}$.

\section{Agradecimentos}

Os autores agradecem a equipe da Embrapa Arroz e Feijão que contribuíram com a execução do experimento, em especial ao Sr. Emerson Moreira dos Santos, pelo suporte fitossanitário. E as empresas que são parceiras do Programa de Melhoramento do Amendoim - Embrapa. Estes resultados estão vinculados ao projeto SEG 20.18.01.021.00. 


\section{Referências}

FERREIRA, D. F. SISVAR: a computer analysis system to fixed effects split plot type designs. Revista Brasileira de Biometria, [S.L.], v. 37, n. 4, p. 529, 20 dez. 2019. http://dx.doi.org/10.28951/rbb.v37i4.450.

HEUERT, J.; SUASSUNA, T. M. F.; MARTINS. K. B. B.; RIBEIRO, T. C. N.; GONÇALVES, W. C. Desempenho agronômico de genótipos de amendoim no estado de Goiás. In: Anais do Encontro Sobre a Cultura do Amendoim, 15., 2018, Jaboticabal. Anais eletrônicos... Campinas: GALOÁ, 2018. Disponível em: <https://proceedings.science/encontro-amendoim2018/papers/desempenho-agronomico-de-genotipos-de-amendoim-noestado-de-goias>. Acesso em: 11 maio 2020.

MARTINS, K. B. B.; RODRIGUES, L. L.; HEUERT, J.; XAVIER, M. F. N.; SUASSUNA, T. M. F.; BETIOL, R. A. B. Desempenho agronômico de novas linhagens de amendoim no Cerrado. In: Anais do Encontro Sobre a Cultura do Amendoim, 16., 2019, Jaboticabal. Anais eletrônicos... Campinas: GALOÁ, 2019. Disponível em: <https://proceedings.science/encontroamendoim-2019/papers/desempenho-agronomico-de-novas-linhagens-deamendoim-no-cerrado>. Acesso em: 15 maio 2020.

MARTINS, K. B. B.; SUASSUNA, T. M. F.; HEUERT, J.; RIBEIRO, R. P. R.; SOAVE, J. H.; SANTOS, L. C. C. Avaliação em genótipos de amendoim tipo runner visando a colheita antecipada. In: Anais do Encontro Sobre a Cultura do Amendoim, 15., 2018, Jaboticabal. Anais eletrônicos... Campinas: GALOÁ, 2018. Disponível em: <https://proceedings.science/encontroamendoim-2018/papers/avaliacao-em-genotipos-de-amendoim-tipo-runnervisando-a-colheita-antecipada>. Acesso em: 15 maio 2020.

RIBEIRO, R. P.; SUASSUNA, T. M. F.; HEUERT, J.; SOAVE, J. H.; SANTOS, L. C. C. Desempenho de genótipos de amendoim na Alta Paulista. In: Anais do Encontro Sobre a Cultura do Amendoim, 15., 2018, Jaboticabal. Anais eletrônicos... Campinas: GALOÁ, 2018. Disponível em: <https://proceedings.science/encontro-amendoim-2018/papers/desempenhode-genotipos-de-amendoim-na-alta-paulista>. Acesso em: 15 maio 2020.

SUASSUNA, T. M. F. et al. 'BRS 421' and 'BRS 423': high oleic peanut cultivars for production in Brazil. Crop Breeding Applied Biotechnology, v. 20, n. 1, e28932018, 2020. https://doi.org/10.1590/1984-70332020v20n1c8

SUASSUNA, T. M. F. et al. BRS 425: the first runner peanut cultivar related to wild ancestral species. Crop Breeding Applied Biotechnology, v. 19, n. 3, p. 373-377, 2019. https://doi.org/10.1590/1984-70332019v19n3c52

SUBRAHMANYAM, P.; MCDONALD, D.; GIBBONS, R. W.; NIGAM, S. N.; NEVILL, D. J. Resistance to rust and late leaf spot diseases in some genotypes of Arachis hypogaea. Peanut Science, v. 9, p. 9-14, 1982. 
Departamento da Agricultura dos Estados Unidos. World Agricultural Producion. Foreign Agricultural Service, wap 5-20, p. 32, mayo 2020.

VIZEU, L. A. Revista Canavieiros - Panorama da cadeia produtiva do amendoim. n. 159, p. 12-13, set. 2019. Disponível em: <https://www.revistacanavieiros.com.br/uploads/pagina/tag/2019/11/e8IGHvh bxTeXEgc9/159-set-baixa.pdf>. Acesso em: 20 abr. 2020. 